\title{
Review of the top 5 cardiology studies of 2013-14
}

\author{
Arden R. Barry, PharmD, ACPR; Jade E. Basaraba, BScPharm, ACPR; Jennifer L. Bong, \\ BScPharm, ACPR; Chloe L. McMillan, BScPharm; Mohamed A. Omar, BScPharm, PhD; \\ Dylan M. Pollmann, BScPharm; Margaret L. Ackman, PharmD, ACPR, FCSHP
}

\section{Introduction}

Within the field of cardiology, many trials are published each year; however, only a portion of these address clinical questions relevant to pharmacists in general practice, and an even smaller number have the potential to change patient care. This article presents 5 recent cardiovascular studies deemed to be the most pertinent to pharmacy practice.

\section{Methods}

The methodology used to determine the studies for review was similar to that conducted for a previous publication. ${ }^{1}$ Cardiology studies deemed relevant to clinical pharmacy practice were identified through discussions with hospital-based pharmacists practising in cardiology. A list of 14 studies published in 201314 was created with accompanying abstracts. This information was included in an online survey emailed to all pharmacists within Alberta Health Services (AHS) (618 clinically deployed pharmacists) and all pharmacists practising at primary care networks within the province of Alberta (approximately 80). The email included a link to the survey, which asked respondents to select up to a maximum of 5 cardiology studies relevant to their practice area. The survey was available for 3 weeks, and 2 reminder emails were sent to AHS pharmacists. Ninety pharmacists responded and the top 5 ranked studies (with 1 honourable mention) are reviewed below.

The selected trials vary in terms of disease state, patient population and therapy investigated. Not unexpectedly, 3 studies involved the use of anticoagulant and/or antiplatelet agents. One trial investigated dabigatran compared to warfarin for the prevention of thromboembolic events in patients with mechanical heart valves. ${ }^{2}$ The other 2 studies focused on patients with coronary artery disease (CAD) after percutaneous coronary intervention (PCI). One trial examined the safety of warfarin and clopidogrel in comparison to triple therapy (acetylsalicylic acid [ASA], clopidogrel and warfarin) in patients requiring long-term oral anticoagulation. ${ }^{3}$ The other investigated the optimal duration of dual-antiplatelet therapy (DAPT) in patients who received PCI with a drug-eluting stent. ${ }^{4}$ Two trials explored heart failure (HF). One addressed spironolactone in the treatment of $\mathrm{HF}$ with preserved ejection fraction, while the second examined an investigational medication known as LCZ-696, which is currently not available in Canada, compared to an angiotensin-converting enzyme inhibitor (ACEI) in patients with HF with reduced ejection fraction. ${ }^{5,6}$ The final study was a retrospective cohort that evaluated 2 thiazide diuretics, hydrochlorothiazide and chlorthalidone, in the outpatient treatment of hypertension. ${ }^{7}$ 


\section{MECHANICAL HEART VALVES}

RE-ALIGN: Dabigatran versus warfarin in patients with mechanical heart valves ( $N$ Engl J Med 2013)

Background: The objective of this multicentre, prospective, randomized, open-label trial was to validate a dabigatran dosing regimen for prevention of thromboembolic complications in patients with mechanical heart valves. ${ }^{2}$

Patients: Included were adult patients who had undergone implantation of a mechanical heart valve within 7 days or more than 3 months before enrollment. Excluded were patients with a creatinine clearance $(\mathrm{CrCl})<40 \mathrm{~mL} / \mathrm{min}$ or those considered at high risk for bleeding. The planned enrollment was 650 patients.

Intervention and control: Patients were randomly assigned (2:1) to receive either dabigatran or warfarin for 12 weeks. Dosing of dabigatran (150$300 \mathrm{mg}$ orally twice daily) was selected based on kidney function and was adjusted to maintain a target predose plasma concentration of $>50 \mathrm{ng} /$ $\mathrm{mL}$. If the dabigatran predose concentration remained subtherapeutic at the highest dose, or if the patient's $\mathrm{CrCl}$ worsened to $<30 \mathrm{~mL} / \mathrm{min}$, patients were switched to warfarin. Dosing of warfarin was adjusted to target an international normalized ratio of 2-3 or 2.5-3.5, according to guideline recommendations and local practice.

Outcomes: The primary end point was the predose plasma level of dabigatran. Additional efficacy and safety outcomes included death, myocardial infarction (MI), stroke or transient ischemic attack (TIA), systemic embolism, valve thrombosis, venous thromboembolism and bleeding.

Results: The trial was prematurely terminated after enrollment of only 252 patients due to an excess of thromboembolic and bleeding events observed in the dabigatran group. The composite of death, stroke or TIA, systemic embolism, or MI occurred in 15 patients (9\%) in the dabigatran group and 4 patients (5\%) in the warfarin group (hazard ratio [HR], 1.94; 95\% confidence interval [CI], 0.64-5.86). Any bleeding occurred in 45 patients $(27 \%)$ in the dabigatran group and 10 patients $(12 \%)$ in the warfarin group (HR, 2.45; 95\% CI, 1.23-4.86).

Implications for practice: Following termination of this trial, Health Canada issued a new contraindication for dabigatran in patients with mechanical heart valves due to the observed increase in harm. ${ }^{8}$ Possible explanations for the increased thromboembolic events with dabigatran include inadequate predose concentrations or the differing mechanisms of action between dabigatran and warfarin. The increase in serious adverse events seen with dabigatran has led to questions regarding the safety of all novel oral anticoagulants (OACs) in this population. Until more data are available, patients with mechanical heart valves should only receive warfarin as antithrombotic therapy. ${ }^{9}$

\section{CORONARY ARTERY DISEASE}

WOEST: Use of clopidogrel with or without aspirin in patients taking oral anticoagulant therapy and undergoing percutaneous coronary intervention: an open-label, randomised, controlled trial (Lancet 2013)

Background: This open-label, multicentre, randomized controlled trial investigated the safety and efficacy of clopidogrel alone, compared to clopidogrel plus ASA, in patients postPCI who were also receiving long-term oral anticoagulation. ${ }^{3}$

Patients: Enrolled were adult patients with a long-term indication for OAC therapy who had a coronary lesion requiring PCI. Exclusion criteria included patients with a history of intracranial bleeding, cardiogenic shock, recent peptic ulcer disease, thrombocytopenia or major bleeding within the past 12 months.

Intervention and control: Patients were randomized to receive clopidogrel $75 \mathrm{mg}$ orally daily plus an OAC (double therapy) or clopidogrel $75 \mathrm{mg}$ orally daily, ASA 80 to $100 \mathrm{mg}$ orally daily and an OAC (triple therapy). Antiplatelet therapy was continued for at least 1 month and up to 1 year for patients receiving bare metal stents for stable CAD and for 1 year or more for patients receiving drug-eluting stents or with an acute coronary syndrome (ACS).

Outcomes: The primary outcome was the occurrence of any bleeding during the 1-year follow-up period. The secondary outcome was the composite of death, MI, stroke, target-vessel revascularization or stent thrombosis. 
Results: A total of 573 patients were enrolled, $80 \%$ of whom were male, with a mean age of 70 years. Atrial fibrillation/flutter was the most common indication for oral anticoagulation (69\%). The proportion of patients who experienced 1 or more bleeding episodes was significantly lower with double therapy compared to triple therapy (19.4\% vs $44.4 \%$; HR, 0.36; 95\% CI, 0.26-0.50). Furthermore, the composite secondary outcome occurred less frequently in the double therapy group (11.1\% vs $17.6 \%$; HR, 0.60; 95\% CI, $0.38-0.94)$. There was no difference between the 2 groups for the individual components of the composite secondary outcome except for all-cause mortality, which was significantly lower with double therapy ( $2.5 \%$ vs $6.3 \%$; HR, 0.39 ; $95 \% \mathrm{CI}$, 0.16-0.93).

Implication for practice: This study provides evidence that double therapy with clopidogrel and an OAC is associated with a lower incidence of bleeding compared to triple therapy (ASA, clopidogrel and OAC) post-PCI. However, the primary end point was mainly driven by a reduction in minor bleeding events. Furthermore, it is not clear why the rate of bleeding events with triple therapy in this trial was considerably higher (44\%) compared to previous registry trials (10\%-15\%). ${ }^{10-12}$ In addition, this study was powered to detect differences in bleeding events rather than rates of stent thrombosis; therefore, it was likely underpowered to detect a difference in this outcome. Finally, the results should not be extrapolated to novel OACs (e.g., apixaban, dabigatran, rivaroxaban) or to newer antiplatelet agents (e.g., prasugrel, ticagrelor), as they were not included in this trial.

DAPT: Twelve or 30 months of dual antiplatelet therapy after drug-eluting stents ( $N$ Engl J Med 2014)

Background: The objective of this multicentre, randomized, double-blind, placebo-controlled trial was to determine if patients who receive PCI with a drug-eluting stent benefit from extendedduration DAPT. ${ }^{4}$

Patients: Randomized patients had received 12 months of open-label DAPT and were event free from death, MI, stroke, repeat revascularization, stent thrombosis and moderate or severe bleeding. Patients also had to also be adherent with DAPT, defined as taking between $80 \%$ and $120 \%$ of prescribed drug. Patients were excluded if they had planned surgery requiring antiplatelet discontinuation for 14 days or more or if they required long-term OAC.

Intervention and control: In addition to ASA 75 to $100 \mathrm{mg}$ orally daily, patients were randomized to a thienopyridine (clopidogrel $75 \mathrm{mg}$ orally daily or prasugrel $10 \mathrm{mg}$ orally daily) or placebo for 18 months.

Outcomes: The co-primary efficacy outcomes were the cumulative incidence of definite or probable stent thrombosis and major adverse cardiovascular and cerebrovascular events (MACCE), defined as the composite of death, MI or stroke. The primary safety outcome was the incidence of moderate or severe bleeding, defined as intracranial hemorrhage, bleeding causing hemodynamic compromise or requiring transfusion.

Results: A total of 9961 patients were randomized. Patients had an average age of 62 years, and $75 \%$ were male. Common indications for PCI were ACS (43\%) and stable angina (38\%). Clopidogrel was used in $65 \%$ of patients. Compared to placebo, extended-duration DAPT significantly reduced MACCE (4.3\% vs 5.9\%; HR, 0.71; 95\% CI, 0.59 0.85 ) and stent thrombosis ( $0.4 \%$ vs $1.4 \%$; HR, 0.29 ; 95\% CI, 0.17-0.48). However, there was a significant increase in non-cardiovascular (CV) death $(1.0 \%$ vs $0.5 \%$; HR, 2.23 ; 95\% CI, 1.32-3.78) and the primary safety end point (2.5\% vs $1.6 \%$; HR, 1.61 ; 95\% CI, 1.21-2.16). A subgroup analysis revealed that the reduction in MACCE was only statistically significant in patients who received first-generation drug-eluting stents (e.g., paclitaxel, sirolimus), which are more prone to stent thrombosis and thus are rarely used in current practice.

Implications for practice: This trial demonstrated that extended-duration DAPT significantly reduced stent thrombosis and MACCE in a lowrisk population; however, this was at the expense of higher rates of moderate and severe bleeding and non-CV death. Consideration should be given to the type of drug-eluting stent that was used, as the same benefit was not observed in the subgroup of patients with newer second-generation stents (e.g., everolimus, zotarolimus). Furthermore, the results should not be applied to patients receiving ticagrelor. The decision to extend the duration of DAPT should not be made at the time of PCI-it should only be considered in patients who are event free and adherent to therapy after 12 months. Some clinicians may choose to extend the duration of DAPT to 30 months in patients with perceived high CV risk and low bleed risk. 


\section{HEART FAILURE}

TOPCAT: Spironolactone for heart failure with preserved ejection fraction ( $N$ Engl J Med 2014)

Background: Previous clinical trials have shown the benefit of mineralocorticoid receptor antagonist therapy (e.g., eplerenone, spironolactone) in reducing mortality and morbidity in patients with $\mathrm{HF}$ with reduced ejection fraction. ${ }^{13-15}$ However, data in patients with HF with preserved ejection fraction are limited. The objective of this multicentre, randomized, double-blind, placebocontrolled trial was to explore the effects of spironolactone in a large population of patients with HF with preserved ejection fraction. ${ }^{5}$

Patients: Included were patients $\geq 50$ years of age with a left ventricular ejection fraction (LVEF) $\geq 45 \%$ and at least 1 sign and symptom of $\mathrm{HF}$. Additional criteria included a serum potassium concentration $<5 \mathrm{mmol} / \mathrm{L}$. Patients were excluded if they had severe renal dysfunction (estimated glomerular filtration rate $[\mathrm{eGFR}]<30 \mathrm{~mL} / \mathrm{min}$ or serum creatinine level $[\mathrm{SCr}] \geq 221 \mu \mathrm{mol} / \mathrm{L}$ ).

Intervention and control: Patients in the intervention group were initiated on spironolactone $15 \mathrm{mg}$ orally daily, which was increased to $45 \mathrm{mg}$ orally daily during the first 4 months after randomization. The control group received matching placebo.

Outcomes: The primary outcome was a composite of death from CV causes, hospitalization for the management of HF or aborted cardiac arrest. The secondary outcomes included all-cause mortality and the individual components of the primary outcome.

Results: A total of 3445 patients were enrolled and followed for a mean of 3.3 years. The median age was 69 years, and $52 \%$ were female. In comparison to placebo, spironolactone did not significantly reduce the primary outcome (18.6\% vs $20.4 \%$; HR, 0.89; 95\% CI, 0.77-1.04). However, the secondary outcome of HF hospitalization was significantly lower in the spironolactone group (12.0\% vs $14.2 \%$; HR, 0.83; 95\% CI, 0.69-0.99). All other secondary outcomes were not statistically significant. Patients in the spironolactone group had a higher rate of hyperkalemia (serum potassium concentration $\geq 5.5 \mathrm{mmol} / \mathrm{L}$ ) compared to placebo ( $18.7 \%$ vs $9.1 \%, p<0.001)$ and were more likely to have renal dysfunction (defined as a doubling of their SCr to a value beyond the upper limit of normal) $(10.2 \%$ vs $7.0 \%, p<0.001)$.
Implications for practice: This study demonstrated that in patients with HF with preserved ejection fraction, spironolactone, as compared to placebo, did not reduce mortality. The reduction in HF hospitalizations is an interesting finding; however, it was a secondary outcome and should not be used to guide practice. Furthermore, spironolactone increased rates of hyperkalemia and renal dysfunction. A regional post hoc analysis showed that patients in Eastern Europe did not benefit from the addition of spironolactone, while a significant benefit was observed in patients in the Americas; nonetheless, this should only be considered hypothesis generating and necessitates further research. The authors of the 2014 Canadian Cardiovascular Society (CCS) HF guidelines suggest that spironolactone could be considered in HF with preserved ejection fraction patients to reduce $\mathrm{HF}$ hospitalizations, although it is classified as a weak recommendation based on low-quality evidence. ${ }^{16}$ As with any indication, patients prescribed spironolactone should have their renal function and serum potassium concentration monitored frequently.

\section{Honourable Mention: PARADIGM-HF:}

Angiotensin-neprilysin inhibition versus enalapril in heart failure ( $N$ Engl J Med 2014)

This prospective, randomized, multicentre, active comparator trial explored the investigational drug LCZ-696 versus enalapril in patients with HF with reduced ejection fraction. ${ }^{6}$ LCZ-696 is classified as an angiotensin receptor-neprilysin inhibitor (ARNI) and is composed of 2 medications: the angiotensin II receptor blocker (ARB) valsartan and sacubitril, a neprilysin inhibitor. Neprilysin is an enzyme responsible for degrading natriuretic peptides and bradykinin. Thus, inhibition counteracts the negative neurohormonal activation that occurs in HF with reduced ejection fraction. Patients with New York Heart Association class II to IV HF symptoms and an LVEF $\leq 40 \%$ were randomized to LCZ-696 $200 \mathrm{mg}$ orally twice daily versus enalapril $10 \mathrm{mg}$ orally twice daily. The trial was discontinued early after 2.3 years, due to benefit observed with LCZ-696. The primary outcome of CV death and HF hospitalization was reduced with LCZ-696 (21.8\% vs $26.5 \%$; HR, 0.80; 95\% CI, 0.73-0.87). LCZ-696 also significantly reduced the individual components of the primary outcome and all-cause mortality. With respect to safety, more symptomatic hypotension was 
observed in the LCZ-696 group. As well, there was a numerically higher incidence of angioedema with LCZ-696, but it did not reach statistical significance (19 vs $10, p=0.13$ ). Elevated SCr, hyperkalemia (serum potassium concentration $>6 \mathrm{mmol} / \mathrm{L}$ ) and cough were significantly higher in the enalapril group. Overall, if approved in Canada, LCZ-696 will likely supersede ACEIs in the treatment of HF with reduced ejection fraction. Based on this study, the CCS has already made a conditional recommendation in the 2014 HF guidelines advocating LCZ-696 over ACEI/ ARBs for the treatment of HF with reduced ejection fraction. ${ }^{16}$ However, little is known about the long-term safety of ARNI therapy. One theoretical concern is an increased risk of cognitive impairment, as neprilysin breaks down amyloid-beta peptide, which is a component of amyloid plaques found in patients with Alzheimer disease.

\section{HYPERTENSION}

Chlorthalidone versus hydrochlorothiazide for the treatment of hypertension in older adults: a population-based cohort study (Ann Intern Med 2013)

Background: The objective of this retrospective population-based cohort study was to compare the effectiveness and safety of chlorthalidone to hydrochlorothiazide (HCTZ) for the treatment of hypertension in older adults. Comparisons between these 2 thiazide diuretics in the published literature have yielded mixed results regarding efficacy and safety. ${ }^{17,18}$ Some clinicians advocate that chlorthalidone should be the thiazide of choice for the treatment of hypertension based on the results of the ALLHAT study. ${ }^{19}$

Patients: Using Ontario health care database information from 1993 to 2010, all patients aged 66 years or older who were newly treated with chlorthalidone or HCTZ and had not been hospitalized for HF, stroke or MI in the prior year were included. As this study was not randomized, there may have been differences in baseline characteristics between the 2 groups. To account for this, each chlorthalidone patient was matched with 1 or 2 patients who received HCTZ based on a propensity score, which matches patients based on similar characteristics such as age, sex and year of treatment initiation.

Intervention and control: Patients who received chlorthalidone or HCTZ at any dose were included if they had not received either drug (or another thiazide) within the previous year. Follow-up was up to a maximum of 5 years.
Outcomes: The primary outcome was a composite of death or hospitalization for MI, HF or ischemic stroke. Safety outcomes of interest were hospitalization for hypokalemia or hyponatremia and all-cause hospitalization.

Results: The cohort consisted of 29,873 patients. The primary outcome occurred in 510 chlorthalidone patients (3.2 events per 100 person-years) and $1265 \mathrm{HCTZ}$ patients $(3.4$ events per 100 person-years) (HR, 0.93; 95\% CI, 0.81-1.06). Furthermore, there was no significant difference in any of the components of the primary outcome. However, patients in the chlorthalidone group were more likely to be hospitalized with hypokalemia (HR, 3.06; 95\% CI, 2.04-4.58) or hyponatremia (HR, 1.68; 95\% CI, 1.24-2.28) as an admission diagnosis.

Implications for practice: Acknowledging the limitations of the retrospective observational design, this study found no significant difference between chlorthalidone and HCTZ with respect to adverse $\mathrm{CV}$ events or death. However, chlorthalidone was more likely to be associated with hypokalemia and hyponatremia requiring hospitalization. Based on these data, it appears as though HCTZ is safer than chlorthalidone in older patients; however, this needs to be confirmed with a large, head-to-head randomized controlled trial. Until more data are available, it is reasonable to suggest that any older adult who is receiving a thiazide diuretic (particularly chlorthalidone) should receive appropriate monitoring for possible electrolyte derangements.

From the Mazankowski Alberta Heart Institute, Pharmacy Services, Alberta Health Services, Edmonton, Alberta.Contact arden.barry@ualberta.ca.

Author Contributions: Dr. Barry initiated the project, was responsible for the design and methodology of identifying the relevant studies, supervised the manuscript writing, contributed to the manuscript by 
writing the summary of the PARADIGM-HF trial, reviewed each draft and revised the final manuscript. Ms. Basaraba contributed to the manuscript by writing the summary of the DAPT trial, Ms. Bong the trial by Dhalla et al, Ms. McMillan the RE-ALIGN trial, Mr. Pollmann the TOPCAT trial and Dr. Omar the WOEST trial. All reviewed the list of relevant studies and revised the final manuscript. Dr. Ackman contributed to the manuscript by writing the introduction and revised the final manuscript.

Declaration of Conflicting Interests: The authors declared no potential conflicts of interest with respect to the research, authorship and/or publication of this article.

Funding: The authors received no financial support for the research, authorship and/or publication of this article.

\section{References}

1. Barry AR, Boswell R, Law EH, et al. Review of the top 5 cardiology studies of 2011-12. Can Pharm J (Ott) 2013;146:213-7.

2. Eikelboom JW, Connolly SJ, Brueckmann M, et al, for the RE-ALIGN Investigators. Dabigatran versus warfarin in patients with mechanical heart valves. $N$ Engl $\mathrm{J} \mathrm{Med}$ 2013;369:1206-14.

3. DeWilde WJ, Oirbans T, Verheuqt FW, et al, for the WOEST study investigators. Use of clopidogrel with or without aspirin in patients taking oral anticoagulant therapy and undergoing percutaneous coronary intervention: an openlabel, randomised, controlled trial. Lancet 2013;381:1107-15. 4. Mauri L, Kereiakes DJ, Yeh RW, et al, for the DAPT Study Investigators. Twelve or 30 months of dual antiplatelet therapy after drug-eluting stents. N Engl J Med 2014;371:2155-66.

5. Pitt B, Pfeffer MA, Assmann SF, et al, for the TOPCAT Investigators. Spironolactone for heart failure with preserved ejection fraction. N Engl J Med 2014;370:1383-92.

6. McMurray JJV, Packer M, Desai AS, et al, for the PARADIGM-HF Investigators and Committees. Angiotensinneprilysin inhibition versus enalapril in heart failure. $N$ Engl J Med 2014:371:993-1004.

7. Dhalla IA, Gomes T, Yao Z, et al. Chlorthalidone versus hydrochlorothiazide for the treatment of hypertension in older adults: a population-based cohort study. Ann Intern Med 2013;158:447-55.

8. Government of Canada. Pradaxa/Pradax (dabigatran etexilate)-new contraindication in patients with prosthetic heart valves requiring anticoagulant treatment due to their valvular status-for health professionals. Available: www.healthycanadians.gc.ca/recall-alert-rappel-avis/ hc-sc/2012/16385a-eng.php (accessed March 12, 2015).

9. Nishimura RA, Otto CM, Bonow RO, et al. 2014 AHA/ ACC guideline for the management of patients with valvular heart disease: a report of the American College of Cardiology/American Heart Association Task Force on Practice Guidelines. J Am Coll Cardiol 2014;63:e57-185.

10. Andrade JG, Deyell MW, Khoo C, et al. Risk of bleeding on triple antithrombotic therapy after percutaneous coronary intervention/stenting: a systematic review and meta-analysis. Can J Cardiol 2013;29:204-12.

11. Karjalainen PP, Porela P, Ylitalo A, et al. Safety and efficacy of combined antiplatelet-warfarin therapy after coronary stenting. Eur Heart J 2007;28:726-32.

12. Lamberts M, Gislason GH, Olesen JB, et al. Oral anticoagulation and antiplatelets in atrial fibrillation patients after myocardial infarction and coronary intervention. J Am Coll Cardiol 2013;62:981-9.

13. Pitt B, Zannad F, Remme WJ, et al, for the Randomized Aldactone Evaluation Study Investigators. The effect of spironolactone on morbidity and mortality in patients with severe heart failure. N Engl J Med 1999;341:709-17.

14. Pitt B, Remme W, Zannad F, et al, for the Eplerenone Post-Acute Myocardial Infarction Heart Failure Efficacy and Survival Study Investigators. Eplerenone, a selective aldosterone blocker, in patients with left ventricular dysfunction after myocardial infarction. $N$ Engl J Med 2003;348:1309-21.

15. Zannad F, McMurray JJ, Krum $\mathrm{H}$, et al, for the EMPHASIS-HF Study Group. Eplerenone in patients with systolic heart failure and mild symptoms. $N$ Engl J Med 2011;364:11-21.

16. Moe G, Ezekowitz J, O'Meara E, et al. The 2014 Canadian Cardiovascular Society heart failure management guidelines focus update: anemia, biomarkers and recent therapeutic trial implications. Can J Cardiol 2015;31:3-16.

17. Rousch GC, Holford TR, Guddati AK. Chlorthalidone compared with hydrochlorothiazide in reducing cardiovascular events: systematic review and network meta-analyses. Hypertension 2012;59:1110-7.

18. van Blijderveen JC, Straus SM, Rodenburg EM, et al. Risk of hyponatremia with diuretics: chlorthalidone versus hydrochlorothiazide. Am J Med 2014;127:763-71.

19. ALLHAT Officers and Coordinators for the ALLHAT Collaborative Research Group. Major outcomes in high-risk hypertensive patients randomized to angiotensin-converting enzyme inhibitor or calcium channel blocker vs diuretic: the Antihypertensive and Lipid-Lowering Treatment to Prevent Heart Attack Trial (ALLHAT). JAMA 2002;288:2981-97. 